\title{
Existence Theorems of Periodic Solutions for Second-Order Nonlinear Difference Equations
}

\author{
Xiaochun Cai ${ }^{1}$ and Jianshe $\mathbf{Y u}^{2}$ \\ ${ }^{1}$ College of Statistics, Hunan University, Changsha, Hunan 410079, China \\ ${ }^{2}$ College of Mathematics and Information Science, Guangzhou University, Guangzhou 510405, China
}

Correspondence should be addressed to Xiaochun Cai, cxchn8@hnu.cn

Received 14 August 2007; Accepted 14 November 2007

Recommended by Patricia J. Y. Wong

The authors consider the second-order nonlinear difference equation of the type $\Delta\left(p_{n}\left(\Delta x_{n-1}\right)^{\delta}\right)+$ $q_{n} x_{n}^{\delta}=f\left(n, x_{n}\right), n \in \mathbb{Z}$, using critical point theory, and they obtain some new results on the existence of periodic solutions.

Copyright (c) 2008 X. Cai and J. Yu. This is an open access article distributed under the Creative Commons Attribution License, which permits unrestricted use, distribution, and reproduction in any medium, provided the original work is properly cited.

\section{Introduction}

We denote by $\mathbb{N}, \mathbb{Z}, \mathbb{R}$ the set of all natural numbers, integers, and real numbers, respectively. For $a, b \in \mathbb{Z}$, define $\mathbb{Z}(a)=\{a, a+1, \ldots\}, \mathbb{Z}(a, b)=\{a, a+1, \ldots, b\}$ when $a \leq b$.

Consider the nonlinear second-order difference equation

$$
\Delta\left(p_{n}\left(\Delta x_{n-1}\right)^{\delta}\right)+q_{n} x_{n}^{\delta}=f\left(n, x_{n}\right), \quad n \in \mathbb{Z},
$$

where the forward difference operator $\Delta$ is defined by the equation $\Delta x_{n}=x_{n+1}-x_{n}$ and

$$
\Delta^{2} x_{n-1}=\Delta\left(\Delta x_{n-1}\right)=\Delta x_{n}-\Delta x_{n-1} .
$$

In (1.1), the given real sequences $\left\{p_{n}\right\},\left\{q_{n}\right\}$ satisfy $p_{n+T}=p_{n}>0, q_{n+T}=q_{n}$ for any $n \in \mathbb{Z}$, $f: \mathbb{Z} \times \mathbb{R} \rightarrow \mathbb{R}$ is continuous in the second variable, and $f(n+T, z)=f(n, z)$ for a given positive integer $T$ and for all $(n, z) \in \mathbb{Z} \times \mathbb{R} .(-1)^{\delta}=-1, \delta>0$, and $\delta$ is the ratio of odd positive integers. By a solution of (1.1), we mean a real sequence $x=\left\{x_{n}\right\}, n \in \mathbb{Z}$, satisfying (1.1).

In $[1,2]$, the qualitative behavior of linear difference equations of type

$$
\Delta\left(p_{n} \Delta x_{n}\right)+q_{n} x_{n}=0
$$


has been investigated. In [3], the nonlinear difference equation

$$
\Delta\left(p_{n} \Delta x_{n-1}\right)+q_{n} x_{n}=f\left(n, x_{n}\right)
$$

has been considered. However, results on periodic solutions of nonlinear difference equations are very scarce in the literature, see $[4,5]$. In particular, in [6], by critical point method, the existence of periodic and subharmonic solutions of equation

$$
\Delta^{2} x_{n-1}+f\left(n, x_{n}\right)=0, \quad n \in \mathbb{Z}
$$

has been studied. Other interesting contributions can be found in some recent papers [7-11] and in references contained therein. It is interesting to study second-order nonlinear difference equations (1.1) because they are discrete analogues of differential equation

$$
\left(p(t) \varphi\left(u^{\prime}\right)\right)^{\prime}+f(t, u)=0 .
$$

In addition, they do have physical applications in the study of nuclear physics, gas aerodynamics, infiltrating medium theory, and plasma physics as evidenced in $[12,13]$.

The main purpose here is to develop a new approach to the above problem by using critical point method and to obtain some sufficient conditions for the existence of periodic solutions of (1.1).

Let $X$ be a real Hilbert space, $I \in C^{1}(\mathbf{X}, \mathbb{R})$, which implies that $I$ is continuously Fréchet differentiable functional defined on $X . I$ is said to be satisfying Palais-Smale condition (P-S condition) if any sequence $\left\{I\left(u_{n}\right)\right\}$ is bounded, and $I^{\prime}\left(u_{n}\right) \rightarrow 0$ as $n \rightarrow \infty$ possesses a convergent subsequence in $X$. Let $B_{\rho}$ be the open ball in $X$ with radius $\rho$ and centered at 0 , and let $\partial B_{\rho}$ denote its boundary.

Lemma 1.1 (mountain pass lemma, see [14]). Let $X$ be a real Hilbert space, and assume that $I \in$ $C^{1}(X, \mathbb{R})$ satisfies the P-S condition and the following conditions:

$\left(\mathrm{I}_{1}\right)$ there exist constants $\rho>0$ and $a>0$ such that $I(x) \geq a$ for all $x \in \partial B_{\rho}$, where $B_{\rho}=\{x \in X$ : $\left.\|x\|_{X}<\rho\right\} ;$

$\left(\mathrm{I}_{2}\right) I(0) \leq 0$ and there exists $x_{0} \bar{\in} B_{\rho}$ such that $I\left(x_{0}\right) \leq 0$.

Then $c=\inf _{h \in \Gamma} \sup _{s \in[0,1]} I(h(s))$ is a positive critical value of I, where

$$
\Gamma=\left\{h \in C([0,1], X): h(0)=0, h(1)=x_{0}\right\} .
$$

Lemma 1.2 (saddle point theorem, see $[14,15]$ ). Let $X$ be a real Banach space, $X=X_{1} \oplus X_{2}$, where $X_{1} \neq\{0\}$ and is finite dimensional. Suppose $I \in C^{1}(\mathbf{X}, \mathbb{R})$ satisfies the P-S condition and

$\left(\mathrm{I}_{3}\right)$ there exist constants $\sigma, \rho>0$ such that $\left.I\right|_{\partial B_{\rho} \cap X_{1}} \leq \sigma$;

$\left(\mathrm{I}_{4}\right)$ there is $e \in \mathbf{B}_{p} \bigcap \mathbf{X}_{1}$ and a constant $\omega>\sigma$ such that $\left.I\right|_{e+X_{2}} \geq \omega$.

Then I possesses a critical value $c \geq \omega$ and

$$
c=\inf _{h \in \Gamma} \max _{u \in B_{\rho} \cap X_{1}} I(h(u)),
$$

where $\Gamma=\left\{h \in C\left(\overline{\mathbf{B}}_{\rho} \cap \mathbf{X}_{1}, \mathbf{X}\right)|h|_{\partial B_{\rho} \cap X_{1}}=i d\right\}$. 


\section{Preliminaries}

In this section, we are going to establish the corresponding variational framework for (1.1).

Let $\Omega$ be the set of sequences

$$
x=\left\{x_{n}\right\}_{n \in \mathbb{Z}}=\left(\ldots, x_{-n}, \ldots, x_{-1}, x_{0}, x_{1}, \ldots, x_{n}, \ldots\right),
$$

that is,

$$
\Omega=\left\{x=\left\{x_{n}\right\}: x_{n} \in \mathbb{R}, n \in \mathbb{Z}\right\} .
$$

For any $x, y \in \Omega, a, b \in \mathbb{R}, a x+b y$ is defined by

$$
a x+b y:=\left\{a x_{n}+b y_{n}\right\}_{n=-\infty}^{+\infty} .
$$

Then $\Omega$ is a vector space. For given positive integer $T, E_{T}$ is defined as a subspace of $\Omega$ by

$$
E_{T}=\left\{x=\left\{x_{n}\right\} \in \Omega: x_{n+T}=x_{n}, n \in \mathbb{Z}\right\} .
$$

Clearly, $E_{T}$ is isomorphic to $\mathbb{R}^{T}$, and can be equipped with inner product

$$
\langle x, y\rangle=\sum_{i=1}^{T} x_{i} y_{i}, \quad \forall x, y \in E_{T}
$$

by which the norm $\|\cdot\|$ can be induced by

$$
\|x\|:=\left(\sum_{i=1}^{T} x_{i}^{2}\right)^{1 / 2}, \quad \forall x \in E_{T} .
$$

It is obvious that $E_{T}$ with the inner product defined by (2.5) is a finite-dimensional Hilbert space and linearly homeomorphic to $\mathbb{R}^{T}$. Define the functional $J$ on $E_{T}$ as follows:

$$
J(x)=\frac{1}{\delta+1} \sum_{n=1}^{T} p_{n}\left(\Delta x_{n-1}\right)^{\delta+1}-\frac{1}{\delta+1} \sum_{n=1}^{T} q_{n} x_{n}^{\delta+1}+\sum_{n=1}^{T} F\left(n, x_{n}\right), \quad \forall x \in E_{T},
$$

where $F(t, z)=\int_{0}^{z} f(t, s) d s$. Clearly, $J \in \mathbf{C}^{1}\left(E_{T}, \mathbb{R}\right)$, and for any $x=\left\{x_{n}\right\}_{n \in \mathbb{Z}} \in \mathbf{E}_{T}$, by using $x_{0}=x_{T}, x_{1}=x_{T+1}$, we can compute the partial derivative as

$$
\frac{\partial J}{\partial x_{n}}=-\Delta\left[p_{n}\left(\Delta x_{n-1}\right)^{\delta}\right]-q_{n} x_{n}^{\delta}+f\left(n, x_{n}\right), \quad n \in \mathbb{Z}(1, T) .
$$

Thus $x=\left\{x_{n}\right\}_{n \in \mathbb{Z}}$ is a critical point of $J$ on $E_{T}$ (i.e., $J^{\prime}(x)=0$ ) if and only if

$$
\Delta\left[p_{n}\left(\Delta x_{n-1}\right)^{\delta}\right]+q_{n} x_{n}^{\delta}=f\left(n, x_{n}\right), \quad n \in \mathbb{Z}(1, T) .
$$

By the periodicity of $x_{n}$ and $f(n, z)$ in the first variable $n$, we have reduced the existence of periodic solutions of (1.1) to that of critical points of $J$ on $E_{T}$. In other words, the functional $J$ is just the variational framework of (1.1). For convenience, we identify $x \in E_{T}$ with $x=\left(x_{1}, x_{2}, \ldots, x_{T}\right)^{T}$. Denote $\mathbf{W}=\left\{\left(x_{1}, x_{2}, \ldots, x_{T}\right)^{T} \in \mathbf{E}_{T}: x_{i} \equiv v, v \in \mathbb{R}, i \in \mathbb{Z}(1, T)\right\}$ and $\mathbf{W}^{\perp}=Y$ such that $\mathbf{E}_{T}=\mathbf{Y} \oplus \mathbf{W}$. Denote other norm $\|\cdot\|_{r}$ on $\mathbf{E}_{T}$ as follows (see, e.g., [16]): $\|x\|_{r}=\left(\sum_{i=1}^{T}\left|x_{i}\right|^{r}\right)^{1 / r}$, for all $x \in \mathbf{E}_{T}$ and $r>1$. Clearly, $\|x\|_{2}=\|x\|$. Due to $\|\cdot\|_{r_{1}}$ and $\|\cdot\|_{r_{2}}$ being equivalent when $r_{1}, r_{2}>1$, there exist constants $c_{1}, c_{2}, c_{3}$, and $c_{4}$ such that $c_{2} \geq c_{1}>0$, $c_{4} \geq c_{3}>0$, and

$$
\begin{aligned}
& c_{1}\|x\| \leq\|x\|_{\delta+1} \leq c_{2}\|x\|, \\
& c_{3}\|x\| \leq\|x\|_{\beta} \leq c_{4}\|x\|,
\end{aligned}
$$

for all $x \in \mathbf{E}_{T}, \delta>0$ and $\beta>1$. 


\section{Main results}

In this section, we will prove our main results by using critical point theorem. First, we prove two lemmas which are useful in the proof of theorems.

Lemma 3.1. Assume that the following conditions are satisfied:

$\left(\mathrm{F}_{1}\right)$ there exist constants $a_{1}>0, a_{2}>0$, and $\beta>\delta+1$ such that

$$
\int_{0}^{z} f(n, s) d s \leq-a_{1}|z|^{\beta}+a_{2}, \quad \forall z \in \mathbb{R}
$$

$\left(\mathrm{F}_{2}\right)$

$$
q_{n} \leq 0, \quad \forall n \in \mathbb{Z}
$$

Then the functional

$$
\mathbf{J}(x)=\frac{1}{\delta+1} \sum_{n=1}^{T} p_{n}\left(\Delta x_{n-1}\right)^{\delta+1}-\frac{1}{\delta+1} \sum_{n=1}^{T} q_{n} x_{n}^{\delta+1}+\sum_{n=1}^{T} F\left(n, x_{n}\right)
$$

satisfies P-S condition.

Proof. For any sequence $\left\{x^{(l)}\right\} \subset E_{T}$, with $J\left(x^{(l)}\right)$ being bounded and $J^{\prime}\left(x^{(l)}\right) \rightarrow 0$ as $l \rightarrow+\infty$, there exists a positive constant $M$ such that $\left|J\left(x^{(l)}\right)\right| \leq M$. Thus, by $\left(\mathrm{F}_{1}\right)$,

$$
\begin{aligned}
-M & \leq J\left(x^{(l)}\right)=\frac{1}{\delta+1} \sum_{n=1}^{T}\left[p_{n}\left(x_{n}^{(l)}-x_{n-1}^{(l)}\right)^{\delta+1}-q_{n}\left(x_{n}^{(l)}\right)^{\delta+1}\right]+\sum_{n=1}^{T} F\left(n, x_{n}^{(l)}\right) \\
& \leq \frac{1}{\delta+1} \sum_{n=1}^{T} p_{n} 2^{\delta+1}\left(\left(x_{n}^{(l)}\right)^{\delta+1}+\left(x_{n-1}^{(l)}\right)^{\delta+1}\right)-\frac{1}{\delta+1} \sum_{n=1}^{T} q_{n}\left(x_{n}^{(l)}\right)^{\delta+1}+\sum_{n=1}^{T} F\left(n, x_{n}^{(l)}\right) \\
& \leq \frac{2^{\delta+1}}{\delta+1} \sum_{n=1}^{T}\left(p_{n}+p_{n+1}\right)\left(x_{n}^{(l)}\right)^{\delta+1}-\frac{1}{\delta+1} \sum_{n=1}^{T} q_{n}\left(x_{n}^{(l)}\right)^{\delta+1}-a_{1} \sum_{n=1}^{T}\left|x_{n}^{(l)}\right|^{\beta}+a_{2} T \\
& =\frac{1}{\delta+1} \sum_{n=1}^{T}\left[2^{\delta+1}\left(p_{n}+p_{n+1}\right)-q_{n}\right]\left(x_{n}^{(l)}\right)^{\delta+1}-a_{1}\left\|x^{(l)}\right\|_{\beta}^{\beta}+a_{2} T .
\end{aligned}
$$

Set

$$
A_{0}=\max _{n \in \mathbb{Z}(1, T)}\left[2^{\delta+1}\left(p_{n}+p_{n+1}\right)-q_{n}\right]
$$

Then $A_{0}>0$. Also, by the above inequality, we have

$$
-M \leq J\left(x^{(l)}\right) \leq \frac{A_{0}}{\delta+1}\left\|x^{(l)}\right\|_{\delta+1}^{\delta+1}-a_{1}\left\|x^{(l)}\right\|_{\beta}^{\beta}+a_{2} T .
$$


In view of

$$
\sum_{n=1}^{T}\left|x_{n}^{(l)}\right|^{\delta+1} \leq T^{(\beta-\delta-1) / \beta}\left(\sum_{n=1}^{T}\left|x_{n}^{(l)}\right|^{\beta}\right)^{(\delta+1) / \beta}
$$

we have

$$
\left\|x^{(l)}\right\|_{\beta}^{\beta} \geq T^{(\delta+1-\beta) /(\delta+1)}\left\|x^{(l)}\right\|_{\delta+1}^{\beta} .
$$

Then we get

$$
-M \leq J\left(x^{(l)}\right) \leq \frac{A_{0}}{\delta+1}\left\|x^{(l)}\right\|_{\delta+1}^{\delta+1}-a_{1} T^{(\delta+1-\beta) /(\delta+1)}\left\|x^{(l)}\right\|_{\delta+1}^{\beta}+a_{2} T .
$$

Therefore, for any $l \in \mathbb{N}$,

$$
a_{1} T^{(\delta+1-\beta) /(\delta+1)}\left\|x^{(l)}\right\|_{\delta+1}^{\beta}-\frac{A_{0}}{\delta+1}\left\|x^{(l)}\right\|_{\delta+1}^{\delta+1} \leq M+a_{2} T .
$$

Since $\beta>\delta+1$, the above inequality implies that $\left\{x^{(l)}\right\}$ is a bounded sequence in $E_{T}$. Thus $\left\{x^{(l)}\right\}$ possesses convergent subsequences, and the proof is complete.

Theorem 3.2. Suppose that $\left(F_{1}\right)$ and following conditions hold:

$\left(\mathrm{F}_{3}\right)$ for each $n \in \mathbb{Z}$,

$$
\lim _{z \rightarrow 0} \frac{f(n, z)}{z^{\delta}}=0
$$

$\left(\mathrm{F}_{4}\right)$

$$
q_{n}<0, \quad \forall n \in \mathbb{Z}(1, T) .
$$

Then there exist at least two nontrivial T-periodic solutions for (1.1).

Proof. We will use Lemma 1.1 to prove Theorem 3.2. First, by Lemma 3.1, $J$ satisfies P-S condition. Next, we will prove that conditions $\left(I_{1}\right)$ and $\left(I_{2}\right)$ hold. In fact, by $\left(\mathrm{F}_{3}\right)$, there exists $\rho>0$ such that for any $|z|<\rho$ and $n \in \mathbb{Z}(1, T)$,

$$
|F(n, z)| \leq-\frac{q_{\max }}{2(\delta+1)} z^{\delta+1}
$$

where $q_{\max }=\max _{n \in \mathbb{Z}(1, T)} q_{n}<0$. Thus for any $x \in E_{T},\|x\| \leq \rho$ for all $n \in \mathbb{Z}(1, T)$, we have

$$
\begin{aligned}
J(x) & \geq-\frac{q_{\max }}{\delta+1} \sum_{n=1}^{T} x_{n}^{\delta+1}+\frac{q_{\max }}{2(\delta+1)} \sum_{n=1}^{T} x_{n}^{\delta+1} \\
& =-\frac{q_{\max }}{2(\delta+1)}\|x\|_{\delta+1}^{\delta+1} \\
& \geq-\frac{q_{\max }}{2(\delta+1)} c_{1}^{\delta+1}\|x\|_{2}^{\delta+1} .
\end{aligned}
$$


Taking $a=-c_{1}^{\delta+1}\left(q_{\max } / 2(\delta+1)\right) \rho^{\delta+1}$, we have

$$
\left.J(x)\right|_{\partial B_{\rho}} \geq a>0
$$

and the assumption $\left(\mathrm{I}_{1}\right)$ is verified. Clearly, $J(0)=0$. For any given $w \in E_{T}$ with $\|w\|=1$ and a constant $\alpha>0$,

$$
\begin{aligned}
J(\alpha w) & =\frac{1}{\delta+1} \sum_{n=1}^{T}\left[p_{n}\left(\alpha w w_{n}-\alpha w_{n-1}\right)^{\delta+1}-q_{n}\left(\alpha w_{n}\right)^{\delta+1}\right]+\sum_{n=1}^{T} F\left(n, \alpha w_{n}\right) \\
& \leq \frac{1}{\delta+1} \sum_{n=1}^{T}\left[p_{n}(2 \alpha)^{\delta+1}-q_{n} \alpha^{\delta+1}\right]-a_{1} \sum_{n=1}^{T}\left|\alpha w_{n}\right|^{\beta}+a_{2} T \\
& \leq \frac{1}{\delta+1} \sum_{n=1}^{T}\left[2^{\delta+1} p_{n}-q_{n}\right] \alpha^{\delta+1} \alpha^{\delta+1}-a_{1} T^{(2-\beta) / 2} \alpha^{\beta}+a_{2} T \\
& \longrightarrow-\infty, \quad(\alpha \longrightarrow+\infty) .
\end{aligned}
$$

Thus we can easily choose a sufficiently large $\alpha$ such that $\alpha>\rho$ and for $\bar{x}=\alpha w \in E_{T}, J(\bar{x})<0$. Therefore, by Lemma 1.1, there exists at least one critical value $c \geq a>0$. We suppose that $\tilde{x}$ is a critical point corresponding to $c$, that is, $J(\tilde{x})=c$, and $J^{\prime}(\tilde{x})=0$. By a similar argument to the proof of Lemma 3.1, for any $x \in E_{T}$, there exists $\widehat{x} \in E_{T}$ such that $J^{\prime}(\widehat{x})=c_{\max }$. Clearly, $\widehat{x} \neq 0$. If $\tilde{x} \neq \hat{x}$, and the proof is complete; otherwise, $\tilde{x}=\hat{x}$ and $c=c_{\max }$. By Lemma 1.1,

$$
c=\inf _{h \in \Gamma} \sup _{s \in[0,1]} J(h(s))
$$

where $\Gamma=\left\{h \in C\left([0,1], E_{T}\right) \mid h(0)=0, h(1)=\bar{x}\right\}$. Then for any $h \in \Gamma, c_{\max }=\max _{s \in[0,1]} J(h(s))$. By the continuity of $J(h(s))$ in $s, J(0) \leq 0$ and $J(\bar{x})<0$ show that there exists some $s_{0} \in$ $(0,1)$ such that $J\left(h\left(s_{0}\right)\right)=c_{\max }$. If we choose $h_{1}, h_{2} \in \Gamma$ such that the intersection $\left\{h_{1}(s) \mid\right.$ $s \in(0,1)\} \cap\left\{h_{2}(s) \mid s \in(0,1)\right\}$ is empty, then there exist $s_{1}, s_{2} \in(0,1)$ such that $J\left(h_{1}\left(s_{1}\right)\right)=$ $J\left(h_{2}\left(s_{2}\right)\right)=c_{\max }$. Thus we obtain two different critical points $x^{1}=h_{1}\left(s_{1}\right), x^{2}=h_{2}\left(s_{2}\right)$ of $J$ in $E_{T}$. In this case, in fact, we may obtain at least two nontrivial critical points which correspond to the critical value $c_{\max }$. The proof of Theorem 3.2 is complete. When $f\left(n, x_{n}\right) \equiv h_{n}$, we have the following results.

Theorem 3.3. Assume that the following conditions hold:

$\left(\mathrm{G}_{1}\right)$

$$
q n<0, \quad \forall n \in \mathbb{Z}(1, T)
$$

$\left(\mathrm{G}_{2}\right)$

$$
\frac{1}{c_{1}^{\delta+1}}\left(\sum_{n=1}^{T} h_{n}^{2}\right)^{(\delta+1) / 2} \sum_{n=1}^{T}\left(-q_{n}\right)<\left(p_{\min } \lambda_{2}^{(\delta+1) / 2}-q_{\max }\right)\left(\sum_{n=1}^{T} h_{n}\right)^{\delta+1}
$$


where $p_{\min }=\min _{n \in \mathbb{Z}(1, T)} p_{n}, q_{\max }=\max _{n \in \mathbb{Z}(1, T)} q_{n}, c_{1}$ is a constant in $(2.10)$, and $\lambda_{2}$ is the minimal positive eigenvalue of the matrix

$$
A=\left(\begin{array}{cccccc}
2 & -1 & 0 & \cdots & 0 & -1 \\
-1 & 2 & -1 & \cdots & 0 & 0 \\
0 & -1 & 2 & \cdots & 0 & 0 \\
\cdots & \cdots & \cdots & \cdots & \cdots & \cdots \\
0 & 0 & 0 & \cdots & 2 & -1 \\
-1 & 0 & 0 & \cdots & -1 & 2
\end{array}\right)_{T \times T}
$$

Then equation

$$
\Delta\left[p_{n} \Delta x_{n-1}\right]^{\delta}+q_{n} x_{n}^{\delta}=h_{n}, \quad n \in \mathbb{Z},
$$

possesses at least one T-periodic solution.

First, we proved the following lemma.

Lemma 3.4. Assume that $\left(G_{1}\right)$ holds, then the functional

$$
J(x)=\frac{1}{\delta+1} \sum_{n=1}^{T} p_{n}\left(\Delta x_{n-1}\right)^{\delta+1}-\frac{1}{\delta+1} \sum_{n=1}^{T} q_{n} x_{n}^{\delta+1}+\sum_{n=1}^{T} h_{n} x_{n}
$$

satisfies P-S condition on $E_{T}$.

Proof. For any sequence $\left\{x^{(l)}\right\} \subset E_{T}$ with $J\left(x^{(l)}\right)$ being bounded and $J^{\prime}\left(x^{(l)}\right) \rightarrow 0$ as $n \rightarrow+\infty$, there exists a positive constant $M$ such that $\left|J\left(x^{(l)}\right)\right| \leq M$. In view of $\left(G_{3}\right)$ and

$$
\sum_{n=1}^{T}\left|h_{n} x_{n}^{(l)}\right| \leq\left(\sum_{n=1}^{T} h_{n}^{2}\right)^{1 / 2}\left(\sum_{n=1}^{T}\left(x_{n}^{(l)}\right)^{2}\right)^{1 / 2},
$$

we have

$$
\begin{aligned}
M & \geq J\left(x^{(l)}\right)=\frac{1}{\delta+1} \sum_{n=1}^{T}\left[p_{n}\left(\Delta x_{n-1}^{(l)}\right)^{\delta+1}\right]-\frac{1}{\delta+1} \sum_{n=1}^{T} q_{n}\left(x_{n}^{(l)}\right)^{\delta+1}+\sum_{n=1}^{T} h_{n} x_{n}^{(l)} \\
& \geq-\frac{1}{\delta+1} \sum_{n=1}^{T} q_{n}\left(x_{n}^{(l)}\right)^{\delta+1}-\sum_{n=1}^{T}\left|h_{n} x_{n}^{(l)}\right| \\
& \geq-\frac{1}{\delta+1} q_{\max } \sum_{n=1}^{T}\left(x_{n}^{(l)}\right)^{\delta+1}-\left(\sum_{n=1}^{T} h_{n}^{2}\right)^{1 / 2}\left(\sum_{n=1}^{T}\left(x_{n}^{(l)}\right)^{2}\right)^{1 / 2} \\
& =-\frac{q_{\max }}{\delta+1}\left\|x^{(l)}\right\|_{\delta+1}^{\delta+1}-\left(\sum_{n=1}^{T} h_{n}^{2}\right)^{1 / 2}\left\|x^{(l)}\right\| \\
& \geq-\frac{q_{\max }}{\delta+1} c_{1}^{\delta+1}\left\|x^{(l)}\right\|^{\delta+1}-\left(\sum_{n=1}^{T} h_{n}^{2}\right)^{1 / 2}\left\|x^{(l)}\right\| .
\end{aligned}
$$

By $\delta+1>1$, the above inequality implies that $\left\{x^{(l)}\right\}$ is a bounded sequence in $E_{T}$. Thus $\left\{x^{(l)}\right\}$ possesses a convergent subsequence, and the proof of Lemma 3.4 is complete. Now we prove Theorem 3.3 by the saddle point theorem. 
Proof of Theorem 3.3. For any $w=(z, z, \ldots, z)^{T} \in W$, we have

$$
J(w)=-\frac{1}{\delta+1} \sum_{n=1}^{T} q_{n} z^{\delta+1}+\sum_{n=1}^{T} h_{n} z .
$$

Take $z=\left(\sum_{n=1}^{T} h_{n} / \sum_{n=1}^{T} q_{n}\right)^{1 / \delta}$ and $\rho=\|w\|=T^{1 / 2}\left|\sum_{n=1}^{T} h_{n} / \sum_{n=1}^{T} q_{n}\right|^{1 / \delta}$, then

$$
J(w)=\frac{\delta}{\delta+1} \frac{\left(\sum_{n=1}^{T} h_{n}\right)^{(\delta+1) / \delta}}{\left|\sum_{n=1}^{T} q_{n}\right|^{1 / \delta}} .
$$

Set

$$
\sigma=\frac{\delta}{\delta+1} \frac{\left(\sum_{n=1}^{T} h_{n}\right)^{(\delta+1) / \delta}}{\left|\sum_{n=1}^{T} q_{n}\right|^{1 / \delta}}
$$

then we have

$$
J(w)=\sigma, \quad \forall w \in \partial B_{\rho} \bigcap Y .
$$

On the other hand, for any $x \in Y$, we have

$$
\begin{aligned}
J(x) & =\frac{1}{\delta+1} \sum_{n=1}^{T} p_{n}\left(\Delta x_{n-1}\right)^{\delta+1}-\frac{1}{\delta+1} \sum_{n=1}^{T} q_{n} x_{n}^{\delta+1}+\sum_{n=1}^{T} h_{n} x_{n} \\
& \geq \frac{p_{\min }}{\delta+1} \sum_{n=1}^{T}\left(\Delta x_{n-1}\right)^{\delta+1}-\frac{q_{\max }}{\delta+1} \sum_{n=1}^{T} x_{n}^{\delta+1}-\sum_{n=1}^{T}\left|h_{n} x_{n}\right| \\
& \geq \frac{p_{\min }}{\delta+1} c_{1}^{\delta+1}\left[\sum_{n=1}^{T}\left(\Delta x_{n-1}\right)^{2}\right]^{(\delta+1) / 2}-\frac{q_{\max }}{\delta+1}\|x\|_{\delta+1}^{\delta+1}-\left(\sum_{n=1}^{T} h_{n}^{2}\right)^{1 / 2}\|x\| \\
& =\frac{p_{\min }}{\delta+1} c_{1}^{\delta+1}\left(x^{T} A x\right)^{(\delta+1) / 2}-\frac{q_{\max }}{\delta+1}\|x\|_{\delta+1}^{\delta+1}-\sum_{n=1}^{T}\left|h_{n} x_{n}\right|,
\end{aligned}
$$

where $x^{T}=\left(x_{1}, x_{2}, \ldots, x_{T}\right)$.

Clearly, $\lambda_{1}=0$ is an eigenvalue of the matrix $A$ and $\xi=(v, v, \ldots, v)^{T} \in E_{T}$ is an eigenvector of $A$ corresponding to 0 , where $v \neq 0, v \in R$. Let $\lambda_{2}, \lambda_{3}, \ldots, \lambda_{T}$ be the other eigenvalues of $A$. By matrix theory, we have $\lambda_{j}>0$ for all $j \in \mathbb{Z}(2, T)$. Without loss of generality, we may assume that $0=\lambda_{1}<\lambda_{2} \leq \cdots \leq \lambda_{T}$, then for any $x \in Y$,

$$
\begin{aligned}
J(x) & \geq \frac{p_{\min }}{\delta+1} c_{1}^{\delta+1} \lambda_{2}^{(\delta+1) / 2}\|x\|^{\delta+1}-\frac{q_{\max }}{\delta+1}\|x\|_{\delta+1}^{\delta+1}-\left(\sum_{n=1}^{T} h_{n}^{2}\right)^{1 / 2}\|x\| \\
& =\left[\frac{p_{\min }}{\delta+1} c_{1}^{\delta+1} \lambda_{2}^{(\delta+1) / 2}-\frac{q_{\max }}{\delta+1} c_{1}^{\delta+1}\right]\|x\|^{\delta+1}-\left(\sum_{n=1}^{T} h_{n}^{2}\right)^{1 / 2}\|x\| \\
& \geq-\frac{\delta}{\delta+1}\left(\sum_{n=1}^{T} h_{n}^{2}\right)^{1 / 2}\left(\frac{\left(\sum_{n=1}^{T} h_{n}^{2}\right)^{1 / 2}}{p_{\min } c_{1}^{\delta+1} \lambda_{2}^{(\delta+1) / 2}-q_{\max } c_{1}^{\delta+1}}\right)^{1 / \delta},
\end{aligned}
$$


as one finds by minimizing with respect to $\|x\|$. That is

$$
J(x) \geq-\frac{\delta}{\delta+1} \frac{\left(\sum_{n=1}^{T} h_{n}^{2}\right)^{(\delta+1) / 2 \delta}\left(1 / c_{1}\right)^{(\delta+1) / \delta}}{\left(p_{\min } \lambda_{2}^{(\delta+1) / 2}-q_{\max }\right)^{1 / \delta}} .
$$

Set

$$
w_{0}=-\frac{\delta}{\delta+1} \frac{\left(\sum_{n=1}^{T} h_{n}^{2}\right)^{(\delta+1) / 2 \delta}\left(1 / c_{1}\right)^{(\delta+1) / \delta}}{\left(p_{\min } \lambda_{2}^{(\delta+1) / 2}-q_{\max }\right)^{1 / \delta}}
$$

then by $\left(G_{2}\right)$, we have

$$
J(x) \geq w_{0}>\sigma, \quad \forall x \in Y .
$$

This implies that the assumption of saddle point theorem is satisfied. Thus there exists at least one critical point of $J$ on $E_{T}$, and the proof is complete. When $q_{n}>0$, we have the following result.

Theorem 3.5. Assume that the following conditions are satisfied:

$\left(\mathrm{G}_{3}\right) 2^{\delta+1}\left[p_{n}+p_{n+1}\right]<q_{n}, q_{n}>0$ for all $n \in \mathbb{Z}(1, T)$;

$\left(\mathrm{G}_{4}\right)\left(\sum_{n=1}^{T} h_{n}^{2}\right)^{(\delta+1) / 2 \delta}\left(\sum_{n=1}^{T} q_{n}\right)^{1 / \delta} C_{1}^{\delta+1}<-A_{0}\left(\sum_{n=1}^{T} h_{n}\right)^{(\delta+1) / \delta}$,

where $A_{0}=\max _{n \in \mathbb{Z}(1, T)}\left[2^{\delta+1}\left(p_{n}+p_{n+1}\right)-q_{n}\right]$.

Then (3.21) possesses at least one T-periodic solution.

Before proving Theorem 3.5, first, we prove the following result.

Lemma 3.6. Assume that $\left(G_{3}\right)$ holds, then $J(x)$ defined by (3.22) satisfies P-S condition.

Proof. For any sequence $\left\{x^{(l)}\right\} \in E_{T}$ with $J\left(x^{(l)}\right)$ being bounded and $J^{\prime}\left(x^{(l)}\right) \rightarrow 0$ as $n \rightarrow+\infty$, there exists a positive constant $M$ such that $\left|J\left(x^{(l)}\right)\right| \leq M$.

Thus

$$
\begin{aligned}
-M & \leq J\left(x^{(l)}\right) \leq \frac{1}{\delta+1} \sum_{n=1}^{T} p_{n}\left(\Delta x_{n-1}^{(l)}\right)^{\delta+1}-\frac{1}{\delta+1} \sum_{n=1}^{T} q_{n}\left(x_{n}^{(l)}\right)^{\delta+1}+\sum_{n=1}^{T} h_{n} x_{n}^{(l)} \\
& \leq \frac{2^{\delta+1}}{\delta+1} \sum_{n=1}^{T}\left(p_{n}+p_{n+1}\right)\left(x_{n}^{(l)}\right)^{\delta+1}-\frac{1}{\delta+1} \sum_{n=1}^{T} q_{n}\left(x_{n}^{(l)}\right)^{\delta+1}+\sum_{n=1}^{T}\left|h_{n} x_{n}^{(l)}\right| \\
& \leq \frac{1}{\delta+1} \sum_{n=1}^{T}\left[2^{\delta+1}\left(p_{n}+p_{n+1}\right)-q_{n}\right]\left(x_{n}^{(l)}\right)^{\delta+1}+\left(\sum_{n=1}^{T} h_{n}^{2}\right)^{1 / 2}\left\|x^{(l)}\right\| \\
& \leq \frac{1}{\delta+1} A_{0}\left\|x^{(l)}\right\|_{\delta+1}^{\delta+1}+\left(\sum_{n=1}^{T} h_{n}^{2}\right)^{1 / 2}\left\|x^{(l)}\right\| \\
& \leq \frac{A_{0}}{\delta+1} c_{2}^{\delta+1}\left\|x^{(l)}\right\|^{\delta+1}+\left(\sum_{n=1}^{T} h_{n}^{2}\right)^{1 / 2}\left\|x^{(l)}\right\| .
\end{aligned}
$$


That is,

$$
-c_{2}^{\delta+1} \frac{A_{0}}{\delta+1}\left\|x^{(l)}\right\|^{\delta+1}-\left(\sum_{n=1}^{T} h_{n}^{2}\right)^{1 / 2}\left\|x^{(l)}\right\| \leq M, \quad \forall n \in \mathbb{N} .
$$

By $\delta+1>1$, the above inequality implies that $\left\{x^{(l)}\right\}$ is a bounded sequence in $E_{T}$. Thus $\left\{x^{(l)}\right\}$ possesses convergent subsequences, and the proof is complete.

Proof of Theorem 3.5. For any $w=(z, z, \ldots, z)^{T} \in \mathbf{W}$, we have

$$
J(\omega)=-\frac{1}{\delta+1} \sum_{n=1}^{T} q_{n} z^{\delta+1}+\sum_{n=1}^{T} h_{n} z
$$

Take $z=\left(\sum_{n=1}^{T} h_{n} / \sum_{n=1}^{T} q_{n}\right), \rho=\|w\|=T^{1 / 2}\left|\sum_{n=1}^{T} h_{n} / \sum_{n=1}^{T}\right|^{1 / \delta}$, then

$$
J(w)=\frac{\delta}{\delta+1} \frac{\left(\sum_{n=1}^{T} h_{n}\right)^{(\delta+1) / \delta}}{\left|\sum_{n=1}^{T} q_{n}\right|^{1 / \delta}}, \quad \forall w \in \partial B_{\rho} \bigcap W
$$

Set

$$
\sigma=\frac{\delta}{\delta+1} \frac{\left(\sum_{n=1}^{T} h_{n}\right)^{(\delta+1) / \delta}}{\left|\sum_{n=1}^{T} q_{n}\right|^{1 / \delta}}
$$

then $J(w)=\sigma$ for all $w \in \partial B_{\rho} \bigcap W$. On the other hand, for any $x \in Y$, we have

$$
\begin{aligned}
J(x) & \leq \frac{1}{\delta+1} \sum_{n=1}^{T}\left[2^{\delta+1}\left(p_{n}+p_{n+1}\right)-q_{n}\right] x_{n}^{\delta+1}+\left(\sum_{n=1}^{T} h_{n}^{2}\right)^{1 / 2}\|x\| \\
& \leq \frac{A_{0}}{\delta+1} c_{2}^{\delta+1}\|x\|^{\delta+1}+\left(\sum_{n=1}^{T} h_{n}^{2}\right)^{1 / 2}\|x\| \\
& \leq-\frac{\delta}{\delta+1}\left(\frac{1}{A_{0}}\right)^{1 / \delta}\left(\frac{1}{c_{2}}\right)^{(\delta+1) / \delta}\left(\sum_{n=1}^{T} h_{n}^{2}\right)^{(\delta+1) / 2 \delta} .
\end{aligned}
$$

Set $w_{0}=-\delta /(\delta+1)\left(1 / A_{0}\right)^{1 / \delta}\left(1 / c_{2}\right)^{(\delta+1) / \delta}\left(\sum_{n=1}^{T} h_{n}^{2}\right)^{(\delta+1) / 2 \delta}$, then $J(x) \leq w_{0}<\sigma$. Thus $-J(x)$ satisfies the assumption of saddle point theorem, that is, there exists at least one critical point of $J$ on $E_{T}$. This completes the proof of Theorem 3.5.

\section{Acknowledgment}

This project is supported by specialized research fund for the doctoral program of higher education, Grant no. 20020532014. 


\section{References}

[1] C. D. Ahlbrandt and A. C. Peterson, Discrete Hamiltonian Systems. Difference Equations, Continued Fractions, and Riccati Equations, vol. 16 of Kluwer Texts in the Mathematical Sciences, Kluwer Academic, Dordrecht, The Netherlands, 1996.

[2] S. S. Cheng, H. J. Li, and W. T. Patula, "Bounded and zero convergent solutions of second-order difference equations," Journal of Mathematical Analysis and Applications, vol. 141, no. 2, pp. 463-483, 1989.

[3] T. Peil and A. Peterson, "Criteria for $C$-disfocality of a selfadjoint vector difference equation," Journal of Mathematical Analysis and Applications, vol. 179, no. 2, pp. 512-524, 1993.

[4] F. Dannan, S. Elaydi, and P. Liu, "Periodic solutions of difference equations," Journal of Difference Equations and Applications, vol. 6, no. 2, pp. 203-232, 2000.

[5] S. Elaydi and S. Zhang, "Stability and periodicity of difference equations with finite delay," Funkcialaj Ekvacioj, vol. 37, no. 3, pp. 401-413, 1994.

[6] Z. M. Guo and J. S. Yu, "The existence of periodic and subharmonic solutions for second-order superlinear difference equations," Science in China Series A, vol. 3, pp. 226-235, 2003.

[7] R. P. Agarwal, Difference Equations and Inequalities: Theory, Methods, and Applications, vol. 228 of Monographs and Textbooks in Pure and Applied Mathematics, Marcel Dekker, New York, NY, USA, 2nd edition, 2000.

[8] R. P. Agarwal and P. J. Y. Wong, Advanced Topics in Difference Equations, vol. 404 of Mathematics and Its Applications, Kluwer Academic, Dordrecht, The Netherlands, 1997.

[9] M. Cecchi, Z. Došlá, and M. Marini, "Positive decreasing solutions of quasi-linear difference equations," Computers \& Mathematics with Applications, vol. 42, no. 10-11, pp. 1401-1410, 2001.

[10] P. J. Y. Wong and R. P. Agarwal, "Oscillations and nonoscillations of half-linear difference equations generated by deviating arguments," Computers \& Mathematics with Applications, vol. 36, no. 10-12, pp. 11-26, 1998, Advances in difference equations, II.

[11] P. J. Y. Wong and R. P. Agarwal, "Oscillation and monotone solutions of second order quasilinear difference equations," Funkcialaj Ekvacioj, vol. 39, no. 3, pp. 491-517, 1996.

[12] M. Cecchi, M. Marini, and G. Villari, "On the monotonicity property for a certain class of second order differential equations," Journal of Differential Equations, vol. 82, no. 1, pp. 15-27, 1989.

[13] M. Marini, "On nonoscillatory solutions of a second-order nonlinear differential equation," Bollettino della Unione Matematica Italiana, vol. 3, no. 1, pp. 189-202, 1984.

[14] P. H. Rabinowitz, Minimax Methods in Critical Point Theory with Applications to Differential Equations, vol. 65 of CBMS Regional Conference Series in Mathematics, American Mathematical Society, Providence, RI, USA, 1986.

[15] J. Mawhin and M. Willem, Critical Point Theory and Hamiltonian Systems, vol. 74 of Applied Mathematical Sciences, Springer, New York, NY, USA, 1989.

[16] K. C. Chang and Y. Q. Lin, Functional Analysis, Peking University Press, Beijing, China, 1986. 